\title{
Occipital Lobe
}

National Cancer Institute

\section{Source}

National Cancer Institute. Occipital Lobe. NCI Thesaurus. Code C12355.

One of the four regions of cortex in each cerebral hemisphere. It is located posterior to the temporal lobe and inferior to the parietal lobe. 